\title{
Non-tuberculous mycobacterial infections in the Scottish Borders: identification, management and treatment outcomes - a retrospective review
}

\author{
${ }^{1} \mathrm{AD}$ McCallum, ${ }^{2} \mathrm{SW}$ Watkin, ${ }^{3} \mathrm{JF}$ Faccenda \\ ${ }^{1}$ Core Medical Trainee 2; ${ }^{2}$ Consultant Physician; ${ }^{3}$ Consultant Physician, Borders General Hospital, Melrose, Roxburghshire, Scotland, UK
}

\begin{abstract}
Non-tuberculous mycobacteria are opportunist pathogens increasingly recognised as a cause of pulmonary and extrapulmonary disease. Treatment is complicated, prolonged and potentially toxic, and due to a limited evidence base, potentially contentious and idiosyncratic. This is a retrospective review of nontuberculous mycobacteria cases in the NHS Borders Health Board between 1992 and 2010. We consider incidence, species identified, drug sensitivity testing and treatment outcome with reference to the British and American Thoracic Society guidelines. Thirty-eight cases of non-tuberculous mycobacteria isolates were identified; $84.21 \%$ were pulmonary and $42.11 \%$ were Mycobacterium avium complex. Incidence rose from $1.92 / 100,000$ in 1993 to $4.43 / 100,000$ in 2010. The British Thoracic Society guidelines were followed in $45.45 \%$ of cases. A total of $36.36 \%$ were successfully treated with another $36.36 \%$ still being treated with antimicrobials. There is a clear need for more research on treatment for this group of 'emerging pathogens' and it remains to be seen if concordance with current guidelines will improve treatment outcomes.
\end{abstract}

KEYWORDS Non-tuberculous mycobacteria, BTS guidelines, epidemiology

DECLARATION OF INTERESTS No conflict of interests declared.
Correspondence to JF Faccenda, Borders General Hospital, Melrose, Roxburghshire, TD6 9BS, Scotland, UK

\section{tel. $+44(0) 1896826000$}

e-mail

jakki.faccenda@borders.scot.nhs.uk

\section{INTRODUCTION}

Non-tuberculous mycobacteria (NTM) are opportunist pathogens ubiquitously found in soil, water, milk, dust and various animals and birds.' While extensive guidelines exist for the management of Mycobacterium tuberculosis complex (MTB) infections (M. tuberculosis, M. africanum, M. bovis) there is a relative paucity of recommendations for the management of NTM infections. Also known as mycobacteria-other-than-tuberculosis (MOTT), opportunist, atypical or environmental mycobacteria, management relies on a limited evidence base. The main recommendations for treating NTM infections are the guidelines published by the British and American Thoracic Societies.'.2

The incidence of NTM infections is increasing in both the immunocompromised and immunocompetent populations and they are being identified more readily with improvements in laboratory methodology, liquid culture techniques and more precise identification. ${ }^{2}$ One study in Ontario, Canada identified a mean increased annual isolation prevalence of $8.4 \%$ for NTM (excluding M. gordonae) between 1997 and 2003, with similar results seen across NTM species. ${ }^{3}$ They concluded that this increase may be in part due to greater isolation sensitivity from the laboratory, increased clinician awareness, and changes in host-pathogen interaction, with increased susceptibility due to immunocompromise or simply advanced age. ${ }^{3}$
Conservation of the 16S rRNA gene in mycobacteria means that mycobacteria can be accurately classed on the basis of minute heterogeneity in this gene rather than relying on phenotypic identification. ${ }^{4}$ Different NTM species have different clinical phenotypes and thus far more than 125 species have been identified. ${ }^{5} \mathrm{~A}$ minority of these NTM species cause the majority of the morbidity, with Mycobacterium avium complex (MAC) causing the bulk of pulmonary NTM disease. ${ }^{5}$ Clinical manifestations include pulmonary disease, skin and soft tissue infections, lymphadenitis and disseminated disease, but NTM may also cause asymptomatic or slowly progressive infection. ${ }^{5}$ NTM disease follows exposure to environmental pathogens rather than human-to-human transmission and as such if a patient has been notified on the basis of a positive smear (pending cultures), contact tracing and chemoprophylaxis for tuberculosis (TB) can be discontinued and the patient 'de-notified' when NTM infection is confirmed. ${ }^{1.6-8}$

Due to the ubiquitous nature of NTM as environmental pathogens, it is important to distinguish between clinical disease and contamination. Chemotherapy is prolonged and is associated with substantial medical costs. ${ }^{9}$ British Thoracic Society (BTS) guidelines propose the significance of an isolate must be considered with respect to the species of NTM, where it was isolated from, the degree of growth and the number of isolates.' Multiple isolates are preferred, particularly when from non-sterile sites. Clinical presentation, appropriate 
exclusion of alternative diagnoses, history of predisposing factors (immunocompromise, pre-existing lung disease etc.), histopathology and radiographic evidence of disease can support diagnosis of NTM infection.' The American Thoracic Society (ATS) concludes that all of the constellation of clinical, radiographic and microbiologic evidence must be met to confirm pulmonary NTM disease. ${ }^{2}$ Both British and American Thoracic Societies acknowledge that limitations in systematic data require that many recommendations are based on expert opinion and that more clinical trials are required. ${ }^{1,2}$ We reflect upon NHS Borders' experience of managing this uncommon but emerging subgroup of infectious diseases.

\section{AIMS}

This audit assesses NTM isolates identified in the Scottish NHS Borders Health Board. We consider the nature of NTM infections isolated in the period 1992 2010 , the species identified, sensitivities to standard antimycobacterial medications, treatment and outcome. Treatment strategies will be compared to the recommendations of the BTS.

\section{METHODOLOGY}

The names of patients with positive mycobacterial isolates were obtained from a database kept by the NHS Borders Microbiology Laboratory over the period I January 1992 to 30 November 2010. This database was established in 1992. Additional names were identified at the monthly local TB group meeting. The database of positive isolates identified by the Scottish Mycobacterial Reference Laboratory was consulted to ensure all relevant potential cases were included. MTB complex (M. tuberculosis, M. africanum, $M$. bovis) or $M$. leprae infections were not included. A case was defined as an individual in whom we had obtained an isolate of nontuberculous mycobacteria.

Patient records were examined for patient age, occupation and address. Details of organism identified, specimen type (sputum, aspirate etc.), location (pulmonary, extra-pulmonary) and sensitivities were collected from the microbiology reports and these data were confirmed by the laboratory. Dates of commencing and completing treatment were noted, the drug regimens used and whether any adverse effects were experienced. Lastly, treatment outcome was recorded and if treatment followed the BTS guidelines.' Where guidelines were absent, the ATS guidelines were consulted. ${ }^{2} \mathrm{New}$ mycobacterial cases were included while culture proceeded and were discounted should they prove to be M. tuberculosis complex. Data for cases still being treated were collected for the database but were not included in the report due to lack of information on outcome of treatment. If cases were known to be immunosuppressed due to HIV or chemotherapy this was documented. If their HIV status was unknown or not specified, cases were assumed to be HIV negative.

Outcomes were recorded as treatment complete (remained well and had no microbiological or radiological evidence of disease on completion of treatment), treatment ongoing or treatment failed (continued microbiological, radiological or clinical evidence of infection despite treatment). Relapse was diagnosed if a previously treatment-complete patient developed symptoms with radiological or microbiological evidence of NTM infection. Patients were considered colonised if multiple positive isolates were found in the absence of clinical illness or active disease. Contamination was diagnosed if only one positive sample was obtained in a clinically well patient with no radiological evidence of infection. Where treatment had been changed on the basis of sensitivities or organism (once identified), the regimen of the longest duration was included.

Samples were processed in the Clinical Pathology Accreditation (CPA) approved Scottish Mycobacteria Reference Laboratory according to the Laboratory Standard Operating Procedures. Auramine phenol staining and microscopy were used for detection of acid-fast bacilli. Primary culture was performed on two Lowenstein-Jensen egg slopes, one containing pyruvate and one containing glycerol as growth supplements and rapid liquid culture (Mycobacterial Growth Indicator Tube; Becton Dickinson) on film positive or precious samples. Other samples were cultured on egg slopes alone. Drug sensitivity testing for slow growing NTM was performed in liquid culture (radiometric BACTEC 460TB; Becton Dickinson). For rapidly growing mycobacteria Etest (AB Biodisk, Sweden) agar diffusion methods were used for drug sensitivity testing. Real time polymerase chain reaction (PCR) was used for rapid detection of MTB complex and NTM in film positive sputa from November 2009. Rapid molecular determination of Mycobacterium tuberculosis complex, rifampicin and isoniazid resistance was determined using Genotype multidrug resistant TB (MDRTB) plus (Hain Lifescience $\mathrm{GmBH}$, Nehren, Germany) from September 2008. The Clinical and Laboratory Standards Intitute recommendations were used in part. We note however that methods have varied over the 18-year period of this review.

\section{RESULTS}

\section{Study characteristics and demographics}

NHS Borders Health Board had a population of 106,764 in the 2001 census. ${ }^{10}$ Table I summarises the characteristics of the NTM cases (patients with a positive NTM isolate) in NHS Borders between 1992 and 2010. A total of 38 cases were identified, of which 
TABLEI Study characteristics and demographics

\begin{tabular}{|l|l|}
\hline Review period & $\begin{array}{c}\text { I January 1992- } \\
\text { 30 November 2010 }\end{array}$ \\
\hline Total NTM cases & $\mathbf{3 8}$ \\
\hline $\begin{array}{l}\text { Current mycobacteria cases } \\
\text { (unclassified) }\end{array}$ & 3 \\
\hline Current NTM cases & 7 \\
\hline Completed treatment NTM cases & 31 \\
\hline Male/Female & $20 / 18$ \\
\hline Pulmonary/Extrapulmonary & $32 / 6$ \\
\hline Mean age (Male/Female) & $64.58(65.8 / 63.22)$ \\
\hline Age range & $7-87$ \\
\hline Case notes unavailable & 8 \\
\hline Case notes destroyed & 13 \\
\hline Immunocompromised patients & 2 \\
\hline
\end{tabular}

seven were still undergoing treatment. $A$ further three cases of mycobacterial infection were identified (as acidfast bacilli) that had not been classified as MTB or NTM and were not included in further analysis. There were 20 male cases, 18 female and the average age was 64.58 years. Thirty-two cases (84.21\%) were pulmonary and only two cases were identified in immunocompromised individuals, both undergoing chemotherapy. Of the pulmonary cases, 26 (81.25\%) cases had NTM cultured from sputum, the remaining six from bronchoalveolar lavage. Three cases had NTM isolated on skin biopsy, one from a Hickmann line blood culture, one from a wrist aspirate and one from a sample of synovial fluid. Due to local policies on retention of medical records after death, I 3 sets of case notes had been destroyed. Eight sets of case notes were unavailable. In these 21 cases species and sensitivity data was available but treatment course and outcome was not. Of the 34 cases where address information was available, all patients were residents of the Borders except one, a 7-year-old girl from Thailand living in Jedburgh. Nine of these cases (25.7I\%) came from Galashiels (with a population of $14,36 \mid$ it is the Borders second largest town ${ }^{10}$ ), seven (20.58\%) from Hawick (the largest Borders town with a population of 14,573), and the remainder from a scattering of smaller Borders towns. There appeared to be no clustering of cases. For the 10 cases with NTM where occupation data was available, two cases with MAC arose in naval engineers, one case with pulmonary M. terrae arose in a 53-year-old woman working in animal welfare, and the remainder from people in a variety of indoor occupations. With such little information available it is not possible to infer any occupational risks. As Figure I demonstrates, NTM isolates have become more common in recent years. In 1993 we recorded 1.92 cases/100,000 population (no cases in 1992) and in 2010,4.43 cases/ 100,000 population using the 2010 population estimates.

\section{Identification of NTM isolates}

The most common NTM isolated was MAC, in 16 (42.11\%) cases. Fourteen of these were pulmonary, one isolated from a wrist aspirate and one from a skin biopsy

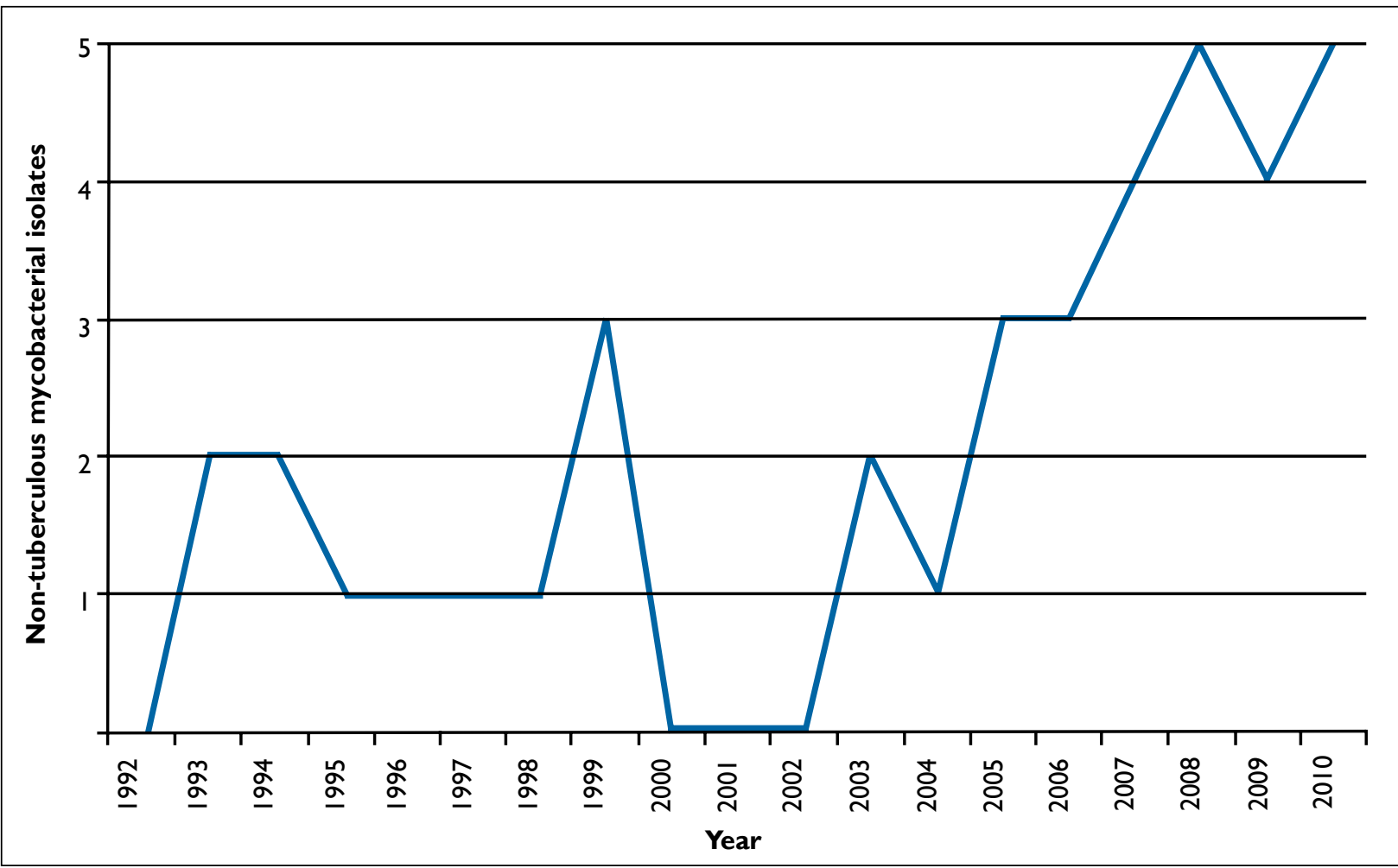

FIGURE I Non-tuberculous isolates by year. 
TABLE 2 Identification of non-tuberculous mycobacterium isolates

\begin{tabular}{|l|l|l|}
\hline $\begin{array}{c}\text { Non-tuberculous } \\
\text { mycobacterium }\end{array}$ & $\begin{array}{c}\text { Number } \\
\text { of cases }\end{array}$ & $\begin{array}{c}\text { Pulmonaryl } \\
\text { Extrapulmonary }\end{array}$ \\
\hline M. avium complex & 16 & $14 / 2$ \\
\hline M. chelonae & 5 & $3 / 2$ \\
\hline M. gordonae & 3 & $3 / 0$ \\
\hline M. kansasii & $\mathrm{I}$ & $\mathrm{I} / 0$ \\
\hline M. malmoense & 5 & $4 / \mathrm{I}$ \\
\hline M. marinum & $\mathrm{I}$ & $0 / 1$ \\
\hline M. nonchromogenicum & 2 & $2 / 0$ \\
\hline M. szulgai & $\mathrm{I}$ & $\mathrm{I} / 0$ \\
\hline M. terrae & 2 & $2 / 0$ \\
\hline M. xenopi & 2 & $2 / 0$ \\
\hline
\end{tabular}

of a facial lesion. Table 2 outlines the numbers of NTM species isolated; ten different species were identified. Of the $15.79 \%$ of extra-pulmonary isolates other than MAC, one case had $M$. marinum isolated from a sample of metacarpophalangeal synovial tissue, one case had $M$. malmoense isolated from an aspirate of an anterior chest wall lesion, one case had $M$. chelonae isolated from Hickmann line cultures and biopsy of skin lesions, and one case had $M$. chelonae isolated from a lower limb skin lesion.

\section{Antimicrobial sensitivity and treatment recommendations}

Table 3 demonstrates the pattern of sensitivity and resistance to common antimicrobials for the 38 cases. Case 2I had M. chelonae isolated from skin biopsy and blood cultures from a Hickmann line. Sensitivity data was only available from the skin biopsy sample. From the other cases, sensitivity data was unavailable for cases 18, 20, 22, $23,24,30$ and 31 . For some this was due to specimens dying on further culture or failing to grow on conventional media. In some of these cases the organism was identified by sequence analysis of the I6S ribosomal rRNA gene but drug sensitivity testing was not performed.

\section{Treatment and outcome}

In many of these NTM cases, empirical anti-tuberculous medications were commenced on identification of acidfast bacilli on microscopy. Where the medications were changed on the basis of sensitivities or identification of organism, we have included the duration of the longest course. Table 4 includes the BTS and ATS treatment guidelines, treatment choice adopted and outcome where known. We also consider whether the BTS guidelines were followed. Unfortunately, data from 21 of the cases on treatment and outcome were unavailable.

Seventeen cases were available for further assessment, of which four had successfully completed treatment (23.53\%) and were now culture negative. Four cases received ongoing treatment $(23.53 \%)$ and one case (case 2 ) was deemed to have failed treatment. There was one relapse of MAC (case 12 - now on lifelong treatment) and case II elected for non-treatment after experiencing general malaise on ethambutol, ciprofloxacin and clarithromycin. Case 28 died before treatment of pulmonary M. malmoense from an unrelated cause. Five cases (29.41\%) were considered to be contaminated or colonised due to the absence of evidence of clinical disease and were not treated - one each of MAC, M. chelonae, M. malmoense, $M$. nonchromogenicum and $M$. terrae. Case 38 (pulmonary $M$. xenopi) was being concurrently treated with posaconazole for probable aspergillosis. Case 21 was identified as being immunosuppressed. He was receiving chemotherapy through a Hickmann line and grew M. chelonae from skin biopsy and Hickmann line blood cultures. The Hickmann line was removed and the skin lesions resolved after 10 weeks of treatment with clarithromycin.

Eleven cases were treated and adherence with BTS treatment guidelines was considered. Of the six cases of MAC, case 2 was changed to the recommended treatment after 12 months of receiving alternative antimicrobials and was deemed to have failed treatment after a total of 54 months. Case 8 was started on appropriate antimicrobials before being discontinued as the patient was deemed to be colonised. Case II did not follow treatment recommendations and did not tolerate treatment. Case 12 received the recommended antimicrobials for only 12 months before relapsing.

The cases with extrapulmonary $M$. chelonae and $M$. malmoense did not follow BTS guidelines but were successfully treated. The case of pulmonary M. xenopi did not follow guidelines and was ongoing. Case 34 of pulmonary $M$. szulgai followed guidelines but as the patient did not tolerate clarithromycin, this was substituted for ciprofloxacin on the basis of sensitivities. No BTS advice exists for the treatment of extrapulmonary M. marinum (case $3 \mathrm{I}$ ) but the ATS guidelines were followed instead to a successful result.

In total, $45.45 \%$ of the treated cases could be considered to have followed the BTS guidelines. Variation existed in terms of duration of therapy and antimicrobials may have been changed on the basis of sensitivity. Of these five cases, one case relapsed, one failed and three were ongoing. Of the six treated cases that did not adhere to BTS guidelines, three were successfully treated, one failed to complete treatment, one was ongoing, and for one, no recommendations existed in the BTS guidelines.

\section{DISCUSSION AND RECOMMENDATIONS}

As the incidence of tuberculosis has declined, NTM have been increasingly recognised as human pathogens. Ubiquitous in nature, NTM may be isolated as contaminants, 
TABLE 3 Antimicrobial sensitivity

\begin{tabular}{|c|c|c|c|c|c|c|c|c|c|c|c|c|c|c|c|c|}
\hline \multirow[b]{2}{*}{ Case } & \multirow[b]{2}{*}{ NTM } & \multirow[b]{2}{*}{ Location } & \multirow[b]{2}{*}{ Culture } & \multirow[b]{2}{*}{ 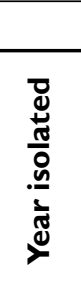 } & \multicolumn{12}{|c|}{ Antimicrobial } \\
\hline & & & & & 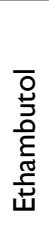 & 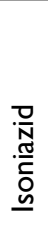 & 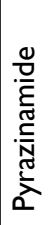 & 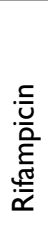 & 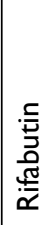 & 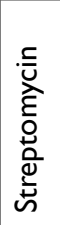 & 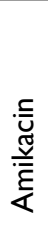 & 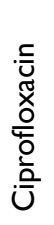 & 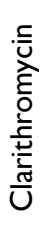 & 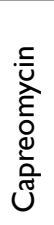 & 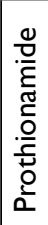 & 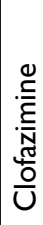 \\
\hline I & M. avium complex & Pulmonary & Sputum & 1993 & $S$ & $\mathrm{R}$ & & $R$ & $R$ & $S$ & $S$ & $\mathrm{R}$ & $S$ & $S$ & $S$ & $S$ \\
\hline 2 & M. avium complex & Pulmonary & Sputum & 1993 & $S$ & $\mathrm{R}$ & & $S$ & $\mathrm{R}$ & & $S$ & $S$ & $S$ & & & \\
\hline 3 & M. avium complex & Pulmonary & BAL & 1994 & $S$ & $R$ & & $R$ & $S$ & & $S$ & $R$ & & & & \\
\hline 4 & M. avium complex & Pulmonary & Sputum & 1994 & $S$ & $\mathrm{R}$ & & $R$ & $\mathrm{R}$ & & $S$ & $S$ & $S$ & & & \\
\hline 5 & M. avium complex & Pulmonary & Sputum & 1996 & $S$ & $R$ & & $R$ & $S$ & & & $R$ & $S$ & & & \\
\hline 6 & M. avium complex & Pulmonary & $\mathrm{BAL}$ & 1999 & $\mathrm{~S}$ & $\mathrm{R}$ & & $R$ & $S$ & & & $\mathrm{~S}$ & $\mathrm{~S}$ & & & \\
\hline 7 & M. avium complex & Pulmonary & BAL & 1999 & $S$ & & & $S$ & $S$ & & & $S$ & $S$ & & & \\
\hline 8 & M. avium complex & Pulmonary & Sputum & 1999 & $S$ & $\mathrm{R}$ & & $R$ & $R$ & & & $S$ & $S$ & & & \\
\hline 9 & M. avium complex & Pulmonary & Sputum & 2003 & $S$ & & & $\mathrm{R}$ & $S$ & & & $S$ & $\mathrm{R}$ & & & \\
\hline 10 & M. avium complex & Pulmonary & Sputum & 2005 & $S$ & $\mathrm{R}$ & & $\mathrm{R}$ & & $\mathrm{R}$ & $\mathrm{R}$ & $\mathrm{R}$ & $S$ & $S$ & $\mathrm{~S}$ & $S$ \\
\hline II & M. avium complex & Pulmonary & BAL & 2007 & $\mathrm{~S}$ & & & $S$ & $\mathrm{~S}$ & & & $S$ & $S$ & & & \\
\hline 12 & M. avium complex & Pulmonary & Sputum & 2007 & $S$ & $\mathrm{R}$ & & $\mathrm{R}$ & $\mathrm{R}$ & & & $S$ & $S$ & & & \\
\hline 13 & M. avium complex & Pulmonary & Sputum & 2009 & $S$ & & & $R$ & $\mathrm{R}$ & $S$ & $S$ & $\mathrm{R}$ & $S$ & $S$ & $S$ & $S$ \\
\hline 14 & M. avium complex & Pulmonary & Sputum & 2010 & $R$ & $\mathrm{R}$ & & $\mathrm{R}$ & $S$ & $\mathrm{R}$ & & $R$ & $S$ & $S$ & $S$ & $S$ \\
\hline 15 & M. avium complex & Face & Skin biopsy & 1995 & $\mathrm{R}$ & $\mathrm{R}$ & $\mathrm{R}$ & $\mathrm{R}$ & $S$ & & $S$ & $\mathrm{R}$ & $S$ & $S$ & $S$ & \\
\hline 16 & M. avium complex & Wrist & $\begin{array}{l}\text { Wrist } \\
\text { aspirate }\end{array}$ & 2010 & $S$ & $\mathrm{R}$ & & $\mathrm{R}$ & $\mathrm{R}$ & $\mathrm{R}$ & $\mathrm{R}$ & $\mathrm{R}$ & & $\mathrm{R}$ & $R$ & \\
\hline 17 & M. chelonae & Pulmonary & Sputum & 2006 & $\mathrm{R}$ & $\mathrm{R}$ & & & & & $\mathrm{R}$ & $\mathrm{R}$ & $S$ & & & \\
\hline 18 & M. chelonae & Pulmonary & Sputum & 2007 & & & & & & & & & & & & \\
\hline 19 & M. chelonae & Pulmonary & Sputum & 2010 & $\mathrm{R}$ & $\mathrm{R}$ & & $\mathrm{R}$ & & $\mathrm{R}$ & $\mathrm{R}$ & $\mathrm{R}$ & $S$ & & & \\
\hline 20 & M. chelonae & Lower limb & Skin biopsy & 2009 & & & & & & & & & & & & \\
\hline 21 & M. chelonae & Skin torso & Skin biopsy & 2010 & $\mathrm{R}$ & & & $\mathrm{R}$ & & $\mathrm{R}$ & $\mathrm{R}$ & $\mathrm{R}$ & $S$ & & & \\
\hline 22 & M. gordonae & Pulmonary & $\mathrm{BAL}$ & 2006 & & & & & & & & & & & & \\
\hline 23 & M. gordonae & Pulmonary & Sputum & 2008 & & & & & & & & & & & & \\
\hline 24 & M. gordonae & Pulmonary & $\mathrm{BAL}$ & 2010 & & & & & & & & & & & & \\
\hline 25 & M. kansasii & Pulmonary & Sputum & 1997 & $\mathrm{R}$ & $\mathrm{R}$ & $\mathrm{R}$ & $\mathrm{R}$ & $\mathrm{R}$ & & & $\mathrm{S}$ & $S$ & & & \\
\hline 26 & M. malmoense & Pulmonary & Sputum & 1998 & $\mathrm{~S}$ & $\mathrm{R}$ & $S$ & $\mathrm{~S}$ & & & & $\mathrm{~S}$ & $S$ & & & \\
\hline 27 & M. malmoense & Pulmonary & Sputum & 2004 & $S$ & $\mathrm{R}$ & $S$ & $S$ & $\mathrm{~S}$ & & & $S$ & $S$ & & & \\
\hline 28 & M. malmoense & Pulmonary & Sputum & 2008 & $S$ & $\mathrm{R}$ & & $S$ & $S$ & & & $S$ & $S$ & & & \\
\hline 29 & M. malmoense & Pulmonary & Sputum & 2008 & $S$ & $\mathrm{R}$ & & $S$ & $S$ & & & $S$ & $S$ & & & \\
\hline 30 & M. malmoense & Chest wall & $\begin{array}{l}\text { Aspirate } \\
\text { chest wall }\end{array}$ & 2005 & & & & & & & & & & & & \\
\hline 31 & M. marinum & $\begin{array}{l}\text { MCPJ } \\
\text { synovitis }\end{array}$ & $\begin{array}{l}\text { Synovial } \\
\text { tissue }\end{array}$ & 2009 & & & & & & & & & & & & \\
\hline 32 & $\begin{array}{l}\text { M. non- } \\
\text { chromogenicum }\end{array}$ & Pulmonary & Sputum & 2005 & $S$ & $\mathrm{R}$ & & $\mathrm{R}$ & $S$ & & & $\mathrm{R}$ & $S$ & & & \\
\hline 33 & $\begin{array}{l}\text { M. non- } \\
\text { chromogenicum }\end{array}$ & Pulmonary & Sputum & 2008 & $S$ & & & $R$ & $\mathrm{R}$ & $\mathrm{R}$ & $\mathrm{R}$ & $\mathrm{R}$ & $S$ & $S$ & $\mathrm{R}$ & $R$ \\
\hline 34 & M. szulgai & Pulmonary & Sputum & 2008 & $S$ & & & S & $S$ & & & $S$ & $S$ & & & \\
\hline 35 & M. terrae & Pulmonary & Sputum & 2006 & $S$ & $\mathrm{R}$ & & $\mathrm{R}$ & $\mathrm{R}$ & & & $\mathrm{R}$ & $S$ & & & \\
\hline 36 & M. terrae & Pulmonary & Sputum & 2007 & $S$ & & & $\mathrm{R}$ & $R$ & $S$ & $S$ & $\mathrm{R}$ & $S$ & $S$ & $\mathrm{R}$ & $S$ \\
\hline 37 & M. xenopi & Pulmonary & Sputum & 2003 & $R$ & & & $S$ & $S$ & & & $S$ & $S$ & & & \\
\hline 38 & M. xenopi & Pulmonary & Sputum & 2009 & $\mathrm{R}$ & $\mathrm{R}$ & & $S$ & $S$ & & & $S$ & $S$ & & & \\
\hline
\end{tabular}


TABLE 4 Treatment and outcome

\begin{tabular}{|c|c|c|c|c|c|c|}
\hline NTM & Case & Treatment & $\begin{array}{l}\text { Duration } \\
\text { (months) }\end{array}$ & $\begin{array}{l}\text { BTS } \\
\text { Guidelines }\end{array}$ & $\begin{array}{l}\text { Adverse } \\
\text { events }\end{array}$ & $\begin{array}{l}\text { Treatment } \\
\text { outcome }\end{array}$ \\
\hline \multirow{9}{*}{$\begin{array}{l}\text { Pulmonary } \\
\text { M. avium }\end{array}$} & \multicolumn{6}{|c|}{ BTS Guidelines: Ethambutol + rifampicin \pm isoniazid - 24 months } \\
\hline & \multicolumn{6}{|c|}{ ATS Guidelines: Ethambutol + rifampicin or azithromycin - until I 2 months culture negative } \\
\hline & \multirow[t]{2}{*}{2} & I. Ison + pyra + rifam & 12 & No & \multirow[t]{2}{*}{ Clari - nausea } & \multirow[t]{2}{*}{ Failure } \\
\hline & & 2. Etham + rifam + cipro + clari & 42 & Yes $^{*}$ & & \\
\hline & 8 & I. Etham + ison + pyra + rifam & 4 & Yes $^{+}$ & Nil & $\begin{array}{l}\text { Stopped - } \\
\text { colonised }\end{array}$ \\
\hline & 11 & I. Etham + cipro + clari & 3 & No & General malaise & Non-completion \\
\hline & \multirow[t]{2}{*}{12} & I. Etham + ison + pyra + rifam & 12 & Yes $^{*}$ & & \multirow{2}{*}{$\begin{array}{l}\text { Relapse - } \\
\text { now life-long } \\
\text { treatment }\end{array}$} \\
\hline & & 2. Etham + rifam + cipro + clari & 18 & Yes $^{*}$ & & \\
\hline & 14 & Etham + rifam & 18 ongoing & Yes & Nil & Ongoing \\
\hline \multirow{3}{*}{$\begin{array}{l}\text { Extra } \\
\text { pulmonary } \\
\text { M. avium }\end{array}$} & \multicolumn{6}{|c|}{ BTS Guidelines: Excision or ethambutol + rifampicin + clarithromycin - 18-24 months } \\
\hline & \multicolumn{6}{|c|}{ ATS Guidelines: Ethambutol + rifampicin + clarithromycin or azithromycin \pm surgical debridement $-6-12$ months } \\
\hline & $\begin{array}{l}16 \\
\text { (wrist) }\end{array}$ & I. Etham + rifam + cipro & 24 ongoing & Yes & Nil & Ongoing \\
\hline \multirow{3}{*}{$\begin{array}{l}\text { Pulmonary } \\
\text { M. chelonae }\end{array}$} & \multicolumn{6}{|c|}{ BTS Guidelines: Ethambutol + rifampicin + clarithromycin \pm surgery - duration unspecified } \\
\hline & \multicolumn{6}{|c|}{ ATS Guidelines: Clarithromycin + second agent on basis of sensitivities - until 12 months culture-negative } \\
\hline & 18 & Nil & N/A & N/A & Nil & $\begin{array}{l}\text { No treatment - } \\
\text { contaminant }\end{array}$ \\
\hline \multirow{4}{*}{$\begin{array}{l}\text { Extra } \\
\text { pulmonary } \\
\text { M. chelonae }\end{array}$} & \multicolumn{6}{|c|}{ BTS Guidelines: Ciprofloxacin + aminoglycoside or imipenem \pm clarithromycin \pm surgical debridement - duration unspecified } \\
\hline & \multicolumn{6}{|c|}{ ATS Guidelines: Clarithromycin + second agent on basis of sensitivities \pm surgery $-4-6$ months } \\
\hline & $\begin{array}{l}20 \\
\text { (lower } \\
\text { limb) }\end{array}$ & I. Etham + rifam & 5 & No & Nil & Complete \\
\hline & $\begin{array}{l}21 \\
\text { (skin } \\
\text { torso) }\end{array}$ & I. Clari & 2.5 & No & Nil & Complete \\
\hline \multirow{4}{*}{$\begin{array}{l}\text { Pulmonary } \\
\text { M. malmoense }\end{array}$} & \multicolumn{6}{|c|}{ BTS Guidelines: Ethambutol + rifampicin - 24 months } \\
\hline & \multicolumn{6}{|c|}{ ATS Guidelines: Ethambutol + isoniazid + rifampicin \pm quinolones \pm macrolides - duration unspecified } \\
\hline & 28 & Died pre-treatment & N/A & N/A & Nil & $\begin{array}{l}\text { No treatment - } \\
\text { died }\end{array}$ \\
\hline & 29 & Nil & N/A & N/A & Nil & $\begin{array}{l}\text { No treatment - } \\
\text { colonised }\end{array}$ \\
\hline \multirow{3}{*}{$\begin{array}{l}\text { Extra } \\
\text { pulmonary } \\
\text { M. malmoense }\end{array}$} & \multicolumn{6}{|c|}{ BTS Guidelines: Ethambutol + rifampicin \pm clarithromycin \pm surgery -24 months } \\
\hline & \multicolumn{6}{|c|}{ ATS Guidelines: Ethambutol + isoniazid + rifampicin \pm quinolones \pm macrolides - duration unspecified } \\
\hline & $\begin{array}{l}30 \\
\text { (chest } \\
\text { wall) }\end{array}$ & I. Rifab + clari & 1.5 & No & Nil & Complete \\
\hline \multirow{3}{*}{$\begin{array}{l}\text { Extra } \\
\text { pulmonary } \\
\text { M. marinum }\end{array}$} & \multicolumn{6}{|c|}{ BTS Guidelines: No recommendations } \\
\hline & \multicolumn{6}{|c|}{ ATS Guidelines: Ethambutol + clarithromycin \pm rifampicin - 2-4 months } \\
\hline & $\begin{array}{l}31 \\
\text { (MCPJ } \\
\text { syno- } \\
\text { vitis) }\end{array}$ & Rifam + clari & 6 & $\mathrm{~N} / \mathrm{A}$ & Nil & Complete \\
\hline \multirow{3}{*}{$\begin{array}{l}\text { Pulmonary } \\
\text { M. nonchrom }\end{array}$} & \multicolumn{6}{|c|}{ BTS Guidelines: No recommendations } \\
\hline & \multicolumn{6}{|c|}{ ATS Guidelines: Macrolide + ethambutol or second agent on basis of sensitivities - duration unspecified } \\
\hline & 32 & Nil & N/A & N/A & Nil & $\begin{array}{l}\text { No treatment - } \\
\text { contaminant }\end{array}$ \\
\hline
\end{tabular}


TABLE 4 (continued) Treatment and outcome

\begin{tabular}{|c|c|c|c|c|c|c|}
\hline NTM & Case & Treatment & $\begin{array}{l}\text { Duration } \\
\text { (months) }\end{array}$ & $\begin{array}{l}\text { BTS } \\
\text { Guidelines }\end{array}$ & $\begin{array}{l}\text { Adverse } \\
\text { events }\end{array}$ & $\begin{array}{l}\text { Treatment } \\
\text { outcome }\end{array}$ \\
\hline \multirow{4}{*}{$\begin{array}{l}\text { Pulmonary } \\
\text { M. szulgai }\end{array}$} & \multicolumn{6}{|c|}{ BTS Guidelines: Ethambutol + rifampicin + clarithromycin - duration unspecified } \\
\hline & \multicolumn{6}{|c|}{ ATS Guidelines: 3-4 anti-tuberculous medications - until 12 months culture-negative } \\
\hline & \multirow[t]{2}{*}{34} & I. Etham + rifam + clari & 4 & Yes & $\begin{array}{l}\text { Clari - } \\
\text { diarrhoea }\end{array}$ & \multirow[t]{2}{*}{ Ongoing } \\
\hline & & 2. Etham + rifam + cipro & 5 ongoing & Yes $^{\dagger+}$ & & \\
\hline \multirow{3}{*}{$\begin{array}{l}\text { Pulmonary } \\
\text { M. terrae }\end{array}$} & \multicolumn{6}{|c|}{ BTS Guidelines: No recommendations } \\
\hline & \multicolumn{6}{|c|}{ ATS Guidelines: Macrolide + ethambutol or second agent on basis of sensitivities - duration unspecified } \\
\hline & 36 & Nil & N/A & N/A & Nil & $\begin{array}{l}\text { No treatment - } \\
\text { contaminant }\end{array}$ \\
\hline \multirow{4}{*}{$\begin{array}{l}\text { Pulmonary } \\
\text { M. xenopi }\end{array}$} & \multicolumn{6}{|c|}{ BTS Guidelines: Ethambutol + rifampicin \pm surgery - 24 months } \\
\hline & \multicolumn{6}{|c|}{$\begin{array}{l}\text { ATS Guidelines: Ethambutol + rifampicin + clarithromycin } \pm \text { surgery OR standard anti-tuberculous therapy + } \\
\text { clarithromycin + quinolones - until I } 2 \text { months culture-negative }\end{array}$} \\
\hline & \multirow[t]{2}{*}{38} & I. Etham + clari + moxi & 5 days & No & Moxi - vomiting & \multirow[t]{2}{*}{ ongoing } \\
\hline & & 2. Etham + cipro + clari & Ongoing & & & \\
\hline \multicolumn{7}{|c|}{$\begin{array}{l}\text { Rifam: rifampicin; Ison: isoniazid; Pyra: pyrazinamide; Etham: ethambutol; Cipro: ciprofloxacin; Clari: clarithromycin; } \\
\text { Rifab: rifabutin; Moxi: moxifloxacin; M. nonchrom: M. nonchromogenicum; MCPJ: metacarpophalangeal joint }\end{array}$} \\
\hline \multicolumn{7}{|c|}{ Yes": treatment according to BTS + other microbial } \\
\hline \multicolumn{7}{|c|}{ Yes $^{+}$: empirical treatment for TB until organism identified. Stopped as recognised as colonisation } \\
\hline Yes $^{++}:$tr & $\mathrm{CCO}$ & to BTS but altered ac & sitivity & & & \\
\hline
\end{tabular}

or from clinical infection,typically in the immunosuppressed. In this study over an 18-year period, 38 NTM cases were identified in the Scottish Borders NHS Board. Incidence increased from $1.92 / 100,000$ in 1993 to $4.43 / 100,000$ in 2010 , and the true incidence of cases in 2010 may be higher still due to as yet unidentified isolates of acid-fast bacilli. This reflects similar trends in England, Wales and Northern Ireland, with 0.9 cases per 100,000 population in 1995, and 2.9 per 100,000 by 2006 ." This may be explained in part by increased recognition of NTM infections as a clinical entity and advances in laboratory techniques including widespread use of liquid culture techniques. Furthermore, increased incidence may reflect changes in the host, pathogen or host-pathogen interaction. Increased susceptibility due to HIV, malignancy and its treatment, pre-existing lung disease or the relative immunodeficiency of advanced age may predispose an individual to NTM infection., ${ }^{2,11,12}$ Furthermore, if crossimmunity arises from $M$. tuberculosis infection or Bacillus Calmette-Guérin (BCG) vaccination, decreases in the prevalence of tuberculosis and BCG uptake may predispose to an increased risk of infection with NTM. ${ }^{13-16}$

Human NTM disease is thought to arise from environmental exposure and no evidence of human-tohuman transmission has been identified to date. ${ }^{2}$ As these cases are non-communicable, statutory reporting is not required, restricting surveillance efforts. More than 100 different species of NTM have been recognised but only $15 \%$ of these are considered pathogenic. ${ }^{7}$ We identified 10 species of NTM in the Borders isolated between 1992 and 2010. Only two cases were known to be immunosuppressed due to chemotherapy, though many were elderly (mean age 64.58 years), predisposing them to a relative immunodeficiency due to ageing. In a retrospective study of cases in England, Wales and Northern Ireland, 53\% of cases were aged 60 or older." NTM are being increasingly recognised in non-HIV patients with pre-existing lung disease, and though none of these patients had cystic fibrosis, chronic obstructive pulmonary disease (COPD) was a common comorbidity.,18-23 In the Netherlands, the increasing notification of MAC has been seen to correlate with an ageing population and increasing prevalence of COPD and was only partly explained by improvements in laboratory identification methods. ${ }^{24}$ Furthermore, this increase in MAC isolation began before improvements in laboratory methods, and other commonly isolated species were not seen to benefit from these improvements. ${ }^{24}$

The Scottish Borders (NHS Board) had a population of 106,764 individuals in the $200 \mathrm{I}$ census compared to a national population of $5,062,011 .{ }^{10}$ Due to the relatively small population, only 38 cases with NTM were identified during this 18-year period. We were unable to infer any occupational risk factors or clustering of cases. The majority of isolates were of pulmonary origin (84.21\%), similar to retrospective studies in the UK and the Netherlands ${ }^{11,25}$ and were most commonly caused by MAC, M. malmoense, $M$. chelonae and $M$. gordonae. The BTS report that $M$. malmoense is the most common NTM in immunocompetent patients in Scotland. It was 
the second most common isolate in this study along with $M$. chelonae, with $13.16 \%$ of cases each. This reflects similar recent findings in NHS Lothian ${ }^{26}$ but differs from the 1995 Lothian study where a cluster of M. malmoense was seen. ${ }^{27}$ The species distribution of our study resembles that seen in the Nijmegen-Arnhem region of the Netherlands ${ }^{25}$ and in Denmark, with MAC the most common species isolated. ${ }^{28}$ This may reflect the increasing prevalence of COPD as seen in the Netherlands. ${ }^{24}$ MAC is more commonly associated with HIVIAIDS but this was not seen in any of these patients.'

Treatment of NTM can be difficult, prolonged and often requires multiple agents. ${ }^{29}$ Distinction between contamination and infection is crucial. To meet the ATS guidelines for diagnosis of NTM pulmonary disease, there must be radiological (chest radiograph or high resolution computed tomography [CT]) and microbiological (positive culture results from at least two expectorated sputum samples or positive culture results from at least one bronchial wash or transbronchial or other lung biopsy with granulomatous inflammation or acid-fast bacilli and positive culture from bronchoscopy or sputum) evidence of infection, combined with exclusion of other disorders such as tuberculosis. ${ }^{2}$ This must be correlated with the clinical picture. The BTS guidelines suggest that the species, the number of isolates, the degree of growth and the identity of the organism should be used to aid diagnosis of clinically significant isolates.' A recent Dutch study found that only $25 \%$ of NTM isolates in the Nijmegen-Arnhem region met the ATS criteria for pulmonary NTM disease, and observed under-treatment (where cases meeting criteria were not treated), overtreatment (where cases not meeting criteria were treated) and use of treatment regimens not proven to be effective. ${ }^{25}$ Similarly, in a Canadian referral centre, $33 \%$ of cases met ATS criteria, and $25 \%$ in a Korean study. ${ }^{3,30}$ Although small, only five of 17 cases in this study were identified as commensals, indicating $70.59 \%$ were considered clinically significant. While this paper considers concordance with the BTS guidelines, adherence to the strict ATS guideline criteria for clinically significant NTM infection may reduce over-treatment in NHS Borders and concurrent risk of unnecessary drug toxicity.

Both the American and British Thoracic Societies concede that due to a paucity of good quality controlled clinical trials, recommendations for treatment of NTM infections often rely on small retrospective studies, a limited evidence base and there is therefore a lack of consensus. ${ }^{1,2}$ As a result of local policies on case record retention, only 17 cases were available for further assessment of treatment and outcome in this study. We identified I I cases that were treated, four of which were deemed to have successfully completed treatment with no further positive cultures and resolution of symptoms. Four had ongoing treatment. Considerable variation in choice and duration of chemotherapy was seen. BTS guidelines were followed in $45.45 \%{ }^{5}$ of the treated cases, for which three were still in treatment, one was deemed a treatment failure and one relapsed. This likely reflects the lack of consensus on treatment of NTM infections, but may also be lower due to some cases being treated before the 1999 publication of the guidelines. Of those six that did not follow BTS guidelines, half were treated successfully. Of the cases with MAC available for further assessment, one treatment failure and one relapse were identified. This is reflected in the literature, with an estimated pulmonary response rate of $50 \%$ and $20 \%$ relapses.' Generally treatment was well tolerated, with two patients suffering nausea or diarrhoea on clarithromycin, one patient experiencing vomiting with moxifloxacin and one patient discontinuing the combination of ethambutol, ciprofloxacin and clarithromycin due to general malaise.

Drug sensitivity testing was carried out on the majority of isolates in this study, although the BTS guidelines conclude that it has little or no benefit in predicting clinical efficacy in NTM infections.' Where resistance has been reported in vitro, synergistic effects may exist in vivo. ${ }^{29,31}$ Furthermore, laboratory breakpoints used to distinguish between sensitivity and resistance to antimicrobials have not been confirmed to be clinically meaningful in the context of NTM. ${ }^{2}$

Taking M. chelonae for further analysis, the three cases with sensitivity data are only found to be sensitive to clarithromycin. The BTS guidelines concede that treatment of pulmonary $M$. chelonae (and other rapidly growing mycobacteria) is difficult and that surgery should be adopted where possible.' Furthermore, the use of quinolones, sulphonamides, amikacin, cefoxitin and imipenem may have a role in treatment.' As a rapidly growing NTM, rifampicin and ethambutol are ineffective and there is no synergy between the agents in this context. ${ }^{2,32}$ Therefore the current BTS recommendations of effective macrolide monotherapy (rifampicin, ethambutol and clarithromycin) for M. chelonae pulmonary disease should be avoided. The ATS suggestion of clarithromycin plus a second agent (on the basis of drug sensitivity testing) is preferable. ${ }^{2}$ The use of clarithromycin alone was seen to be successful in treating one case with M. chelonae skin disease (case 21). Of the cases of MAC, all but two were sensitive to ethambutol but the majority were resistant to both rifampicin and isoniazid. Current BTS guidelines recommend the use of a combination of ethambutol, rifampicin and isoniazid for a period of 24 months', adopted for some of the cases in this study. A subsequent multicentre randomised controlled trial by the BTS has suggested that the addition of isoniazid to rifampicin and ethambutol reduces failure rates but may be associated with a higher death rate. ${ }^{33}$ Case 12 was treated with this regimen for 12 months before relapsing and commencing lifelong treatment with alternative chemotherapeutic agents on the basis of sensitivities. 
Even in the more rare NTM infections, with limited trial data the clinical utility of drug sensitivity testing is unclear. We would conclude that the value of drug sensitivity testing is very limited and that the BTS and ATS guidelines should be referred to for choice of therapy. Ideally, experienced clinicians should be consulted prior to initiation of therapy.

In conclusion, this study demonstrates that NTM isolates have increased in the NHS Borders region over the last 18 years. These cases arise predominantly in the elderly, are mostly pulmonary and may be associated with other comorbidities. Considerable variation in treatment strategies was found. Following the ATS guidelines for identifying clinically relevant NTM isolation may reduce unnecessary and prolonged treatment. The rarity of NTM infections renders good quality clinical trials difficult, but with the growing recognition of NTM as 'emerging pathogens' we would hope to see NTM infections as an increasing focus of research. Multicentre trials and creation of regional referral centres may improve management of this difficult group of diseases.

\section{Acknowledgements}

We would like to thank Dr lan Laurenson at the Scottish Mycobacteria Reference Laboratory for his assistance. We would also like to thank Dr Edward James and the staff at the Borders General Hospital Laboratory for their assistance in data collection.

\section{REFERENCES}

I Subcommittee of the Joint Tuberculosis Committee of the British Thoracic Society. Management of opportunistic mycobacterial infections: Joint Tuberculosis Committee guidelines 1999. Thorax 2000; 55:210-18. http://dx.doi.org//0.1 I36/thorax.55.3.210

2 Griffith DE, Aksamit T, Brown-Elliot BA et al. An official ATS/IDSA statement: diagnosis, treatment, and prevention of nontuberculous mycobacterial diseases. Am J Respir Crit Care Med 2007; 175:367416. http://dx.doi.org/I0.II64/rccm.200604-57IST

3 Marras TK, Chedore P, Ying AM et al. Isolation prevalence of pulmonary non-tuberculous mycobacteria in Ontario, 1997-2003. Thorax 2007;62:66 I-66.http://dx.doi.org// 0.1 I 36/thx.2006.070797

4 Patel JB, Leonard DGB, Pan $X$ et al. Sequence-based identification of Mycobacterium species using the MicroSeq 500 I6S rDNA Bacterial Identification System.J Clin Microbiol 2000; 38:246-5I.

5 Johnson MM, Waller EA, Leventhal JP. Non-tuberculous mycobacterial pulmonary disease. Curr Opin Pulm Med 2008; 14:203-10. http://dx.doi.org//0.1097/MCP.0b0 I 3e3282f9e650

6 Bange FC, Brown BA, Smaczny $C$ et al. Lack of transmission of mycobacterium abscessus among patients with cystic fibrosis attending a single clinic. Clin Infect Dis 200I; 32:1648-50. http:// dx.doi.org// 0.1086/320525

7 Kobashi Y, Yoshida K, Niki Y et al. Sibling cases of mycobacterium avium complex disease associated with hematological disease. J Infect Chemother 2006; 12:331-34. http://dx.doi.org//0.1007/ s10156-006-046I-z

8 Olivier KN, Weber DJ, Wallace RJ Jr et al. Nontuberculous mycobacteria. I: multicenter prevalence study in cystic fibrosis. Am J Respir Crit Care Med 2003; 167:828-34. http://dx.doi.org//0.1 I64/ rccm.200207-6780C

9 Leber A, Marras TK. The cost of medical management of pulmonary nontuberculous mycobacterial disease in Ontario, Canada. Eur Respir J 20 10; 37: I 158-65. http://dx.doi.org//0.1 I83/0903 I936.000550I0

10 General Register Office for Scotland [Internet]. Comparative population profile: Borders Health Board Scotland [cited 2010 Dec 18]. Available from: http://www.scrol.gov.uk/scrol/browser/ profile.jsp?profile=Population\&mainLevel=HealthBoard\&mainArea =Borders\&mainText=\&mainTextExplicitMatch=null\&compLevel= CountryProfile\&compText=\&compTextExplicitMatch=null

II Moore JE, Kruijshaar ME, Ormerod LP. Increasing reports of nontuberculous mycobacteria in England,Wales and Northern Ireland, 1995-2006. BMC Public Health 2010; 10:612. http://dx.doi. org/I0.I I86/I47I-2458-I0-6/2

12 Gruver AL, Hudson LL, Sempowski GD. Immunosenescence of ageing. J Pathol 2007; 21 I:144-56. http://dx.doi.org/I0.1002/path.2104

I3 Ahn CH, Lowell JR, Onstad GD et al. A demographic study of disease due to Mycobacterium kansasii or M. intracellulare-avium in Texas. Chest 1979; 75: I 20-25. http://dx.doi.org// 0.1378/chest.75.2.120

14 Fogan L. PPD antigens and the diagnosis of mycobacterial disease. Arch Intern Med 1969; 124:49-54. http://dx.doi.org//0.100I/ archinte. I24.I.49

15 Trnka L, Danková D, Svandová E. Six years' experience with the discontinuation of BCG vaccination: 4. Protective effect of BCG vaccination against the Mycobacterium avium intracellulare complex. Tuber Lung Dis 1994; 75:348-52. http://dx.doi. org/10.1016/0962-8479(94)90080-9

16 Romanus V, Hallander HO, Wåhlén P et al. Atypical mycobacteria in extrapulmonary disease among children. Incidence in Sweden from 1969 to 1990, related to changing BCG-vaccination coverage. Tuber Lung Dis 1995; 76:300-10. http://dx.doi.org//0.1016/S0962-8479(05)80028-0

17 Raviglione M. Diseases due to other mycobacteria. In: Heymann D, editor. Control of communicable diseases manual. Washington: American Public Health Association; 2004. p.572-73.

18 Henriques B, Hoffner SE, Petrini B et al. Infection of Mycobacterium malmoense in Sweden: Report of 221 cases. Clin Infect Dis 1994; 18:596-600. http://dx.doi.org//0.1093/clinids//8.4.596

19 Henry MT, Inamdar L, O'Riordain D et al. Nontuberculous mycobacteria in non-HIV patients: epidemiology, treatment and response. Eur Respir J 2004; 23:74I-46. http://dx.doi.org/10.1 I83/0 9031936.04.00II4004

20 Lamden K, Watson J, Knerer G et al. Opportunist mycobacteria in England and Wales 1982-1994. Commun Dis Rep CDR Review 1996; 6: 147-51.

2I McGarvey J, Bermudez LE. Pathogenesis of nontuberculous mycobacterial infections. Clin Chest Med 2002; 23:569-83. http:// dx.doi.org/I0.1016/S0272-523I(02)000I2-6

22 O'Brien RJ, Geiter LJ, Snider DE Jr. The epidemiology of nontuberculous mycobacterial diseases in the United States: results from a national survey. Am Rev Respir Dis 1987; 135: I007-I4.

23 Prince DS, Peterson DD, Steiner RM et al. Infection with Mycobacterium avium complex in patients without predisposing conditions. N Engl J Med 1989; 321:863-68. http://dx.doi. org/I0.1056/NEJMI989092832/I304

24 van Ingen J, Hoefsloot W, Dekhuijzen PN et al. The changing pattern of clinical Mycobacterium avium isolation in the Netherlands. Int J Tuberc Lung Dis 2010; 14: I 176-80.

25 van Ingen J, Bendien SA, de Lange WC et al. Clinical relevance of non-tuberculous mycobacteria isolated in the Nijmegen-Arnhem region, The Netherlands. Thorax 2009; 64:502-6. http://dx.doi. org/10.1136/thx.2008.110957 
26 Ching, Y. Assessment of management and response to treatment of nontuberculous mycobacteria infection. Unpublished data 2007.

27 Böllert FGE, Watt B, Greening AP et al. Non-tuberculous pulmonary infections in Scotland: a cluster in Lothian? Thorax 1995; 50: 188-90. http://dx.doi.org/I0.1 I36/thx.50.2.188

28 Thomsen VO, Andersen AB, Miörner $H$. Incidence and clinical significance of non-tuberculous mycobacteria isolated from clinical specimens during a 2-y nationwide survey. Scand J Infect Dis 2002; 34:648-53. http://dx.doi.org//0.1080/003655402/0I478/3

29 Banks J, Jenkins PA. Combined versus single antituberculous drugs on the in vitro sensitivity patterns of non-tuberculous mycobacteria. Thorax 1987; 42:838-42. http://dx.doi.org//0.I I36/thx.42.II.838

30 Koh WJ, Kwon OJ, Jeon K. Clinical significance of nontuberculous mycobacteria isolated from respiratory specimens in Korea. Chest 2006; | 29:34 |-48. http://dx.doi.org/ /0.| 378/chest. I 29.2.34 |
31 Heifets LB. Synergistic effect of rifampicin, streptomycin, ethionamide and ethambutol on Mycobacterium intracellulare. Am Rev Respir Dis 1982; 125:43-48.

32 Swenson JM, Thornsberry C, Silcox VA. Rapidly growing mycobacteria: testing of susceptibility to 34 antimicrobial agents by broth microdilution. Antimicrob Agents Chemother 1982; 22: 186-92.

33 Research Committee of the British Thoracic Society. First randomised controlled trial of treatments for pulmonary disease caused by $M$. avium intracellulare, $M$. malmoense and $M$. xenopi in HIV negative patients: rifampicin, ethambutol and isoniazid versus rifampicin and ethambutol. Thorax 200I; 56:167-72. http://dx.doi. org/10.1136/thorax.56.3.167

\section{Exclusive offer for RCPE Collegiate Members $\mathbf{5 0 \%}$ discount on Fellowship subscriptions}

We are pleased to offer a significant discount in annual subscription rates for eligible Collegiate Members who are Consultants and wish to progress to Fellowship. ${ }^{*}$ Collegiate Members of four years' standing who are successful in their nomination for Fellowship will be able to obtain a $50 \%$ discount on their first year's Fellowship subscription and a $25 \%$ reduction on their second year's subscription, offering savings of up to $£ 300$.

Please access details of our reduced subscription rates, including concessionary elements for Fellows working less than halftime or on maternity leave, at: www.rcpe.ac.uk/join/ fellowshipoffer.php

\section{Fellowship confers a range of additional} benefits and opportunities:

- International peer and public recognition through the use of the 'FRCP Edin' postnominals.

- Professional support for revalidation/ recertification.

- The opportunity to participate in projects and working groups to determine the future direction of education and clinical medicine.

- The opportunity to help maintain national and international clinical standards by acting as an MRCP(UK) examiner.

- The opportunity to inform College responses to external policy consultations in your specialty.

- The opportunity to participate in the governance of the College through election to committees and to Council.

*Eligible candidates should normally have held a substantive Consultant post or equivalent for at least 11 months. If you hold such a post and are interested in being considered for Fellowship, the principal method is nomination by an existing Fellow. Please e-mail Avril Harries at a.harries@rcpe.ac.uk for a list of Fellows in your area. Alternatively, you may wish to discuss nomination with one of our Regional Advisers or consider self-nomination. 